\title{
The risk of endothelial and erectile dysfunctions in Behçet's disease: a comparative analysis of mucocutaneous and systemic patient groups
}

\author{
Yasemin Erdem ${ }^{1}$, Selcuk Erdem², Mehmet Barburoglu³, Ekrem Bilal Karaayvaz ${ }^{4}$, Erkingul Shugaiv ${ }^{5}$, Murat Kurtuncu ${ }^{5}$, \\ Artur Salmaslioglu ${ }^{3}$, Huseyin Oflaz ${ }^{4}$, Afet Akdag Kose ${ }^{6}$, Ates Kadioglu ${ }^{2}$
}

\author{
${ }^{1}$ Department of Dermatology, Health Science University, Sisli Hamidiye Etfal Training and Research Hospital, Istanbul, Turkey \\ ${ }^{2}$ Department of Urology, Faculty of Medicine, Istanbul University, Istanbul, Turkey \\ ${ }^{3}$ Department of Radiology, Faculty of Medicine, Istanbul University, Istanbul, Turkey \\ ${ }^{4}$ Department of Cardiology, Faculty of Medicine, Istanbul University, Istanbul, Turkey \\ ${ }^{5}$ Department of Neurology, Faculty of Medicine, Istanbul University, Istanbul, Turkey \\ ${ }^{6}$ Department of Dermatology and Venereology, Faculty of Medicine, Istanbul University, Istanbul, Turkey
}

Adv Dermatol Allergol 2021; XXXVIII (4): 622-628

DOI: https://doi.org/10.5114/ada.2021.108931

\begin{abstract}
Introduction: Behçet's disease (BD) is a chronic inflammatory disease which can be limited to only mucocutaneous tissues or can affect different systems of the body.

Aim: To investigate the association of endothelial and erectile dysfunctions with $\mathrm{BD}$, on the basis of comparative analysis between mucocutaneous and systemic BD.

Material and methods: Thirty-eight men diagnosed with BD were included in the present study. The patients were stratified into two groups as mucocutaneous BD ( $n=20$, MBD group), and systemic BD ( $n=18$, SBD group). Erectile dysfunction (ED) was assessed using the Erectile Function domain of the International Index of Erectile Function (IIEF-EF) questionnaire. The coronary flow reserve (CFR) assessment was done for analysing endothelial dysfunction (EnD), and CFR $<2$ was defined as EnD. Penile Doppler ultrasonography (PDU) was performed for ED. The demographic and clinical parameters, IIEF-EF score ED classification, CFR and PDU test findings were compared between two groups.

Results: The median age was 34 (22-52) years in the overall population, and there was no difference between two groups $(p=0.558)$. Time from diagnosis was significantly longer ( 24 vs. 102 months, $p=0.021)$ and the use of immunosuppressive therapies was higher ( 0 vs. $70.6 \%, p<0.001$ ) in the SBD group. In overall, median CFR was 1.92 (1.1-5.96), and there was no difference between two groups (1.88 vs. 1.97, $p=0.812$ ). The percentage of patients with CFR $<2$ was similar in two groups ( $52.6 \%$ vs. $52.9 \%, p=0.985)$. The ED status according to IIEF-EF was similar in two groups ( $45 \%$ vs. $27.8 \%, p=0.538$ ) as well as according to PDU analyses ( $10 \%$ vs. $16.7 \%, p=0.544)$.

Conclusions: The increased risk of endothelial, and erectile dysfunctions should be considered in men who were diagnosed with mucocutaneous and systemic BD.
\end{abstract}

Key words: Behçet's disease, endothelial dysfunction, erectile dysfunction, mucocutaneous, systemic.

\section{Introduction}

Behçet's disease (BD) is a chronic inflammatory disorder characterized by recurrent oral, and genital ulcers, uveitis and skin lesions [1]. Mucocutaneous lesions in BD (oral ulcers, genital ulcers, erythema nodosum-like lesions, papulopustular lesions, superficial thrombophlebitis or other cutaneous lesions) are the common presen- tation types of the disease and the patients are initially diagnosed by these lesions [2]. In a great proportion of $\mathrm{BD}$ patients, the disease generally affects only the skin and/or mucosa without any systemic involvement during patients' life. However, it may also affect the vascular, neurological, gastrointestinal, musculoskeletal or urinary systems, and the eye [1-3]. When compared to the pa-

Address for correspondence: Yasemin Erdem MD, Department of Dermatology, Health Science University, Sisli Hamidiye Etfal Training and Research Hospital, Etfal sok. Sisli, Istanbul, 34371, Turkey, phone: +90 212 3735000, fax: +90 2122240772 ,

e-mail: erdemyasemin1@gmail.com

Received: 21.11.2019, accepted: 16.03.2020. 
tients with systemic involvement, the need for complex treatment and mortality risk is reduced and the prognosis is better in the patients whose disease affects only the skin and/or mucosa.

Vascular BD (VBD) is characterized by the involvement of vessels $[4,5]$. The prevalence of VBD varied between $14.3 \%$ and $40 \%$ in several reports, and young men were reported to have an increased frequency and risk of severity for $\operatorname{VBD}[4,5]$. Although veins and arteries in any size may be affected by the disease, VBD predominantly affects the venous system and common manifestations of VBD are superficial thrombophlebitis and deep-vein thrombosis of lower extremities [4]. However, neurological BD (NBD) is another common manifestation of systemic disease, with a prevalence of $5-10 \%[6,7]$. In NBD, both the central and peripheral nervous system may be affected by the disease. The prognosis of NBD is relatively worse, particularly in patients with dural sinus thrombosis or central parenchymal involvement [6, 7].

On the other hand, erectile dysfunction (ED), one of the most common problem of men's sexual health, is described as the persistent inability to attain or maintain an erection sufficient to permit satisfactory sexual performance [8]. The prevalence of ED varies between $19.2 \%$ and $65.6 \%$ in men aged 30-80 years in different countries [9]. The penile erection consists of arterial dilation, trabecular smooth muscle relaxation and activation of the corporeal veno-occlusive system and it is coordinated by several hormonal, neurological and vascular factors [10]. As any disease affecting these factors may cause ED, endothelial dysfunction (EnD) is attributed to be the prominent key factor in the ED pathophysiology. However, BD is also suggested to be a systemic vasculitis in which EnD plays a role as an underlying factor in pathophysiology of the disease [11]. From this point of view, EnD should play a role as a common pathophysiological factor in male BD patients with ED.

The association between ED, and systemic (vascular or neurological) BD was previously reported in some limited studies $[12,13]$. In the present study, on the basis of EnD, we aimed to investigate the prevalence of ED in mucocutaneous BD (MBD) patients compared to systemic BD (SBD) patients.

\section{Material and methods}

\section{Patients}

Thirty-eight men diagnosed with BD were included in the present study. The diagnosis, and treatment of the patients were established and maintained in the Dermatology or Neurology Department at our institution. All patients fulfilled the International Study Group Criteria for BD at the time of diagnosis [14]. The patients were stratified into two groups as mucocutaneous BD $(n=20$, MBD group), and systemic BD ( $n=18$, SBD group).

In the MBD group, the disease affected only the skin and/or mucosa with no vascular, neurological or other systemic involvement. The patients in this group presented with only mucocutaneous lesions such as oral/genital ulcers, erythema nodosum-like lesions, papulopustular lesions, and the pathergy test was positive in all patients.

In the SBD group, 7 patients had vascular involvement, and 11 had neurological involvement. Six patients with vascular involvement presented with deep venous thrombosis at lower extremities and the other 1 patient with vascular involvement presented with superior vena cava thrombosis. The central nervous system was affected in all 11 patients with neurological involvement.

After the demographics (age, body mass index (BMI)), and clinical (smoking history, oral aphthae, genital ulcer, other skin lesions, the positive pathergy and Human Leukocyte Antigen-B51 (HLA-B51) tests, family history, deep vein thrombosis, pulmonary artery aneurysm, vena cava superior and inferior thrombosis, time from the first symptom, time from diagnosis, treatment type) parameters were recorded in the Dermatology or Neurology Departments, the patients were referred to the Urology Department for ED assessment. The Erectile Function domain of the International Index of Erectile Function (IIEF-EF) score was questioned for each patient. The questions in IIEF-EF, and ED scoring system were detailed in the Supplementary Table S1. Common risk factors of erectile and endothelial dysfunctions were also investigated for defining the exclusion criteria. The patients with diabetes mellitus, hypertension (systolic blood pressure > $140 \mathrm{~mm} \mathrm{Hg}$ and/or diastolic blood pressure $>90 \mathrm{~mm} \mathrm{Hg}$ ), atherosclerotic vascular disease, dyslipidaemia (total cholesterol and/or triglyceride levels > $240 \mathrm{mg} / \mathrm{dl}$ and > $160 \mathrm{mg} / \mathrm{dl}$ in fasting plasma), chronic renal failure, thyroid disease, history of pelvic or prostatic surgery, central or peripheral neurological disease and hormonal abnormalities were excluded from the study. Accordingly, blood samples were collected from each patient to measure serum glucose $(\mathrm{mg} / \mathrm{dl})$, hemoglobin- $\mathrm{A}_{1 \mathrm{c}}\left(\mathrm{HbA}_{1 \mathrm{c}} \%\right.$ \%), triglycerides (mg/dl), total cholesterol $(\mathrm{mg} / \mathrm{dl})$, testosterone $(\mathrm{g} / \mathrm{ml})$ and folliclestimulating hormone ( $\mathrm{FSH}, \mathrm{mlU} / \mathrm{ml}$ ) levels.

After ED assessment in the Urology Department, the patients were referred to the Cardiology Department for Coronary Flow Reserve (CFR) Assessment to identify EnD.

\section{Coronary flow reserve assessment}

In this non-invasive diagnostic technique, CFR recordings were performed with the Vivid 7 echocardiography device (General Electric, USA) using a middle-range-frequency $(3-8 \mathrm{MHz}$ ) broadband transducer in the left anterior descending (LAD) coronary artery by transthoracic colour Doppler echocardiography. The coronary blood in the mid-to-distal LAD coronary artery was examined by colour Doppler flow mapping guidance with the optimal velocity range $(+12$ to $+15 \mathrm{~cm} / \mathrm{s})$. Then, the sample volume (1.5 or $2.0 \mathrm{~mm}$ wide) was positioned on the colour signal in the LAD coronary artery. Variables of the LAD coronary artery velocity were measured using the fast 
Fourier transformation analysis. After baseline recordings of flows, dipyridamole $(0.56 \mathrm{mg} / \mathrm{kg}$; Persantine, Boehringer Ingelheim) was infused over a 4-min period. An additional infusion of dipyridamole $(0.28 \mathrm{mg} / \mathrm{kg}$ over a 2-min period) was used unless the heart rate a $10 \%$ increase from the baseline. Two minutes after the end of the infusion, hyperaemic spectral profiles in the LAD coronary artery were recorded. All images were recorded for playback analysis, and were later measured off-line. Average diastolic peak flow velocity (ADPV) and the average mean diastolic flow velocity were measured at baseline and under hyperaemic conditions. CFR was defined as the ratio of ADPV at hyperaemia to ADPV at baseline. CFR $\geq 2.0$ was considered normal in the present study.

Finally, the patients were referred to the Radiology Department for Penile Doppler Ultrasonography (USG) test to quantitatively determine whether the patients have ED or not.

\section{Penile Doppler ultrasonography}

Colour Doppler Ultrasonography was carried out with a LOGIQ 7 Ultrasound Machine 10.0 MHz transducer. Cavernosal arteries were identified along their entire course from the crus to the apex of the penis and their diameters were measured. After the initial ultrasound examination, $2 \mathrm{ml}$ papaverine- $\mathrm{HCl}$ (60 mg) was injected into the corpora cavernosa. The arterial diameters of both cavernosal arteries were measured at the mid-shaft after 5 min of the intracavernous injection. Peak systolic velocities (PSV) and end diastolic velocities (EDV) of cavernosal arteries were measured three times at the mid shaft after 5, 10 and 15 min of the intracavernous injection. We accepted arterial failure criteria as the cavernous artery two times PSV less than $30 \mathrm{~cm} / \mathrm{s}$, and the sum of the percentage of the dilation of both cavernous arteries less than $70 \%$. We accepted venous failure criteria as the cavernous artery two times EDV more than $5 \mathrm{~cm} / \mathrm{s}$.

This study was approved by clinical study local ethics committee of the Istanbul University, Istanbul Faculty of Medicine (2012/423-981), and informed consent was obtained from all patients in this study.

\section{Statistical analysis}

The comparative analyses were done using the Pearson's $\chi^{2}$ test for categorical variables. KolmogorovSmirnov test was used for normality analysis. Since the distribution of all continuous variables were not normal, Mann-Whitney $U$ test was used for non-parametric continuous variables. Categorical variables were listed using the number, and percentage value, whereas non-parametric continuous variables were defined using median, minimum and maximum values. The statistical significance was defined as $p<0.05$.

\section{Results}

The median age of the overall patients was 34 (2252) years. All patients had oral aphthae, $92 \%$ had genital ulcers, and $73 \%$ had a positive pathergy test. HLA-B51 positivity and family history were detected in $60 \%$ and $23 \%$ of the patients, respectively. The median time from the first symptom was 120 (12-324) months, and the median time from diagnosis was 60 (6-128) months. The general characteristics, and clinical findings of the patients are summarized in Table 1, while the comparison of MBD and SBD groups in terms of demographic data and clinical features are summarized in Table 2 . The median age ( 36 vs. 33.5 years, $p=0.558)$, BMI (24.03 vs. $25.05 \mathrm{~kg} / \mathrm{m}^{2}, p=0.273$ ), the levels of serum glucose (89 vs. $90.5 \mathrm{mg} / \mathrm{dl}, p=0.608), \mathrm{HbA}_{1 \mathrm{c}}(5.4 \mathrm{vs} .5 .3 \%, p=0.369)$, triglycerides (117 vs. $126.5 \mathrm{mg} / \mathrm{dl}, p=0.447$ ), total cholesterol (126.5 vs. $118 \mathrm{mg} / \mathrm{dl}, p=0.907)$, testosterone (3.86 vs. $3.30 \mathrm{~g} / \mathrm{ml}, p=0.313$ ) and FSH (2.95 vs. $4.15 \mathrm{mIU} / \mathrm{ml}$, $p=0.077$ ) levels and smoking history (65.0\% vs. $72.2 \%$, $p=0.632$ ) were found to be similar between the MBD and SBD groups. No statistically significant difference was observed between two groups in terms of oral aphthae, genital ulcers, pathergy positivity, HLA-B51 positivity, and BD family history. The time from the first symptom (84 vs. 138 months, $p=0.107$ ) and the time from diagnosis (24 vs. 102 months, $p=0.021$ ) were higher in the SBD group compared to the MBD group and this difference was statistically significant for time from diagnosis ( $p=0.021$. We found that none of the MBD patients used immunosuppressive agents, while 12 (70.6\%, with no knowledge regarding immunotherapy use for 1 patient) patients in the SBD group received immunosuppressive agents (azathioprine in 9 patients, mycophenolate mofetil in 1 patient, infliximab in 1 patient and corticosteroid in 1 patient).

The median CFR values were below 2 in both groups and there was no statistically significant difference between the two groups (1.88 vs. 1.97, $p=0.812$ ). Coronary flow reserve assessment could not be performed for $1 \mathrm{pa}-$ tient in each group. Endothelial dysfunction (CFR $<2$ ) was determined in $10(52.6 \%)$ of the 19 patients in the MBD group, and in 9 (52.9\%) of the 17 patients in the SBD group, and this comparison was statistically nonsignificant ( $p=0.985)$ (Table 3).

In the evaluation of ED according to IIEF-EF score, 14 (36.8\%) of the sexually active patients described as mild, moderate or severe ED. Nine (45.0\%) patients in the MBD group, and 5 (27.8\%) patients in the SBD group defined ED according to IIEF-EF score, and this comparison was statistically non-significant $(p=0.538)$. Penile Doppler USG evaluation detected ED in a total of 5 (13.2\%) patients. Three (16.7\%) of these patients were in the SBD group and remaining 2 (10\%) patients were in the MBD group. There was no statistically significant difference 
Table 1. The descriptive lists of demographic and clinical parameters of overall population and subgroups

\begin{tabular}{|c|c|c|c|c|}
\hline \multirow[t]{2}{*}{ Parameter } & \multirow[t]{2}{*}{ Overall } & \multirow[t]{2}{*}{ Mucocutaneous } & \multicolumn{2}{|c|}{ Systemic } \\
\hline & & & Vascular & Neurological \\
\hline$n$ & 38 & 20 & 7 & 11 \\
\hline Age [years] & $34(22-52)$ & $36(22-52)$ & $35(29-29)$ & $32(28-47)$ \\
\hline Body mass index [kg/m²] & $24.74(17.92-34.76)$ & $24.03(17.92-33.33)$ & $25.24(21.12-34.76)$ & $24.86(21.60-28.19)$ \\
\hline Smoking history $(n, \%)$ & $26,68.4$ & $13,65.0$ & $5,71.4$ & $8,72.7$ \\
\hline Serum glucose level [mg/dl] & $89(54-136)$ & $89(74-107)$ & $88(71-136)$ & $93(54-116)$ \\
\hline Serum $\mathrm{HbA}_{1 \mathrm{c}}$ level (\%) & $5.4(4.6-6.2)$ & $5.4(4.9-5.8)$ & $4.9(4.6-6.2)$ & $5.3(4.7-6.0)$ \\
\hline Serum triglyceride level [mg/dl] & $123.5(46-213)$ & $117(46-213)$ & $112(71-205)$ & $132(56-203)$ \\
\hline Serum cholesterol level [mg/dl] & $128(97-152)$ & $126.5(97-139)$ & $116.5(97-149)$ & $124(102-152)$ \\
\hline Serum testosterone level $[\mathrm{g} / \mathrm{ml}]$ & $3.73(1.22-10.62)$ & $3.86(1.22-10.62)$ & $3.48(3.10-6.12)$ & $3.04(2.04-5.67)$ \\
\hline Serum FSH level $[\mathrm{mlU} / \mathrm{ml}]$ & $4.14(1.3-9.6)$ & $2.95(1.3-9.6)$ & $4.3(2.3-9.2)$ & $4.0(2.9-9.3)$ \\
\hline Oral aphthae $(n, \%)$ & 38,100 & 20,100 & 7,100 & 11,100 \\
\hline Genital ulcer $(n, \%)$ & $35,92.1$ & 20,100 & $6,85.7$ & $9,81.8$ \\
\hline Other skin lesions* $(n, \%)$ & $29,76.3$ & $15,75.0$ & $5,71.4$ & $9,81.8$ \\
\hline Pathergy test $(n, \%)$ & $28,73.7$ & $16,80.0$ & $4,57.1$ & $8,72.7$ \\
\hline HLA-B51 positivity $(n, \%)$ & $23,60.5$ & $11,55.0$ & $5,71.4$ & 7, 63.6 \\
\hline Family history $(n, \%)$ & $9,23.7$ & $5,25.0$ & $1,14.3$ & $3,27.3$ \\
\hline Deep vein thrombosis $(n, \%)$ & $7,23.3$ & - & 7,100 & - \\
\hline Pulmonary artery aneurysm $(n, \%)$ & - & - & - & - \\
\hline Vena cava superior and inferior thrombosis $(n, \%)$ & $1,2.7$ & - & $1,14.3$ & - \\
\hline Time from the first symptom [months] & $120(12-324)$ & $84(12-324)$ & $144(48-216)$ & $120(48-228)$ \\
\hline Time from diagnosis [months] & $60(1-228)$ & $24(1-228)$ & $60(2-168)$ & $120(36-228)$ \\
\hline \multicolumn{5}{|l|}{ Treatment": } \\
\hline Immunosuppressive agents ( $n, \%)$ & $12,32.4$ & 0,0 & $1,16.7$ & $11,100.0$ \\
\hline Non-immunosuppressive therapy $(n, \%)$ & $25,67.6$ & $20,100.0$ & $5,83.3$ & 0,0 \\
\hline
\end{tabular}

between the two groups in terms of ED determined by Penile Doppler USG ( $p=0.544)$ (Table 3).

Five patients who were found to have ED in Penile Doppler USG assessment were detailed in Table 4. Accordingly, arterial insufficiency was detected in 3 patients whereas veno-occlusive insufficiency was detected in 2 patients. Endothelial dysfunction (CFR $<2$ ) was found in $4(80 \%)$ of these patients.

\section{Discussion}

Behçet's disease is a syndrome which affects different organs, and systems. Although the prevalence is similar in both sexes, BD is known to be more severe in young men. Accordingly, the presence of EnD and ED in the male population of BD was investigated in the present study. The incidence of self-reported ED using IIEF-EF score was $36.8 \%$ in overall patients, $45.0 \%$ in the MBD group and $27.8 \%$ in the SBD group. Penile Doppler USG findings showed that the ED rate was $13.2 \%$ in overall patients and it was $10 \%$ and $16.7 \%$ in the MBD and SBD groups, respectively. These findings showed that the ED risk in $\mathrm{BD}$ is also increased in the mucocutaneous group regardless of systemic involvement. To our knowledge, there is no study in the current literature about ED risk in MBD. In previous studies, the ED incidence was reported to increase in vascular and neurological BD patients $[12,13]$. Erdoğru et al. reported ED prevalence as 63\% in 19 male NBD patients. The penile Doppler USG evaluation in their study showed that 11 of 12 (91.7\%) ED detected patients had vascular ED (7 patients had mixed (arterial + venous), 2 patients had arterial, 2 patients had venous) and 1 patient had neurological ED. The authors stated that ED in NBD is caused by EnD and inflammation in the vascular system rather than by neurological factors [12]. In another study, ED was reported in 2 VBD patients [13]. 
Table 2. The comparison of demographic and clinical parameters between mucocutaneous and systemic groups

\begin{tabular}{|c|c|c|c|}
\hline Parameter & Mucocutaneous & Systemic & $P$-value \\
\hline$n$ & 20 & 18 & \\
\hline Age [years] & $36(22-52)$ & $33.5(28-47)$ & 0.558 \\
\hline Body mass index $\left[\mathrm{kg} / \mathrm{m}^{2}\right]$ & $24.03(17.92-33.33)$ & $25.05(21.12-34.76)$ & 0.273 \\
\hline Smoking history $(n, \%)$ & $13,65.0$ & $13,72.2$ & 0.632 \\
\hline Serum glucose [mg/dl] & $89(74-107)$ & $90.5(54-136)$ & 0.608 \\
\hline Serum $\mathrm{HbA}_{1 \mathrm{c}}(\%)$ & $5.4(4.9-5.8)$ & $5.3(4.6-6.2)$ & 0.369 \\
\hline Serum triglyceride level [mg/dl] & $117(46-213)$ & $126.5(56-203)$ & 0.447 \\
\hline Serum cholesterol level [mg/dl] & 126.5 (97-139) & $118(97-152)$ & 0.907 \\
\hline Serum testosterone level $[\mathrm{g} / \mathrm{ml}]$ & $3.86(1.22-10.62)$ & $3.30(2.04-6.12)$ & 0.313 \\
\hline Serum FSH level [mIU/ml] & $2.95(1.3-9.6)$ & $4.15(2.3-9.3)$ & 0.077 \\
\hline Oral aphthae $(n, \%)$ & 20,100 & 18,100 & - \\
\hline Genital ulcer $(n, \%)$ & 20,100 & $15,83.3$ & 0.057 \\
\hline Pathergy test $(n, \%)$ & $16,80.0$ & $12,66.7$ & 0.351 \\
\hline HLA-B51 positivity $(n, \%)^{*}$ & $11,64.7$ & $12,75.0$ & 0.520 \\
\hline Family history $(n, \%)$ & $5,25.0$ & $4,22.2$ & 0.841 \\
\hline Time from the first symptom [months] & $84(12-324)$ & $138(48-228)$ & 0.107 \\
\hline Time from diagnosis [months] & $24(1-228)$ & $102(2-228)$ & 0.021 \\
\hline Immunosuppressive therapy $(n, \%)^{\#}$ & 0,0 & $12,70.6$ & $<0.001$ \\
\hline
\end{tabular}

Table 3. The comparative analyses of endothelial and erectile dysfunction between mucocutaneous and systemic groups

\begin{tabular}{|c|c|c|c|c|}
\hline Parameter & Overall & Mucocutaneous & Systemic & $P$-value \\
\hline$n$ & 38 & 20 & 18 & \\
\hline Coronary flow reserve & $1.92(1.1-5.96)$ & $1.88(1.21-5.96)$ & $1.97(1.1-2.94)$ & 0.812 \\
\hline Endothelial dysfunction $(n, \%)^{*}$ & $19,52.8$ & $10,52.6$ & $9,52.9$ & 0.985 \\
\hline Erectile dysfunction ( $n, \%)$ & $5,13.2$ & 2,10 & $3,16.7$ & 0.544 \\
\hline \multicolumn{5}{|l|}{ Penile Doppler ultrasonography: } \\
\hline Median peak systolic velocity-Right CA $[\mathrm{cm} / \mathrm{s}]$ & $45(9-113)$ & $45.5(28-91)$ & $41.0(9-113)$ & 0.248 \\
\hline Median peak systolic velocity-Left CA [cm/s] & $43.5(9-82)$ & $43.5(28-82)$ & $43.5(9-70)$ & 0.895 \\
\hline Median end diastolic velocity-Right CA [cm/s] & $-4(-8-13)$ & $-5.0(-8.0-9.0)$ & $-3.5(-6-13)$ & 0.241 \\
\hline Median end diastolic velocity-Left CA [cm/s] & $-4(-9-13)$ & $-4.0(-9-10)$ & $-4.5(-8-13)$ & 0.496 \\
\hline \multicolumn{5}{|l|}{ IIEF score: } \\
\hline Normal $(n, \%)$ & $20,52.6$ & $9,45.0$ & $11,61.1$ & 0.538 \\
\hline ED-mild, moderate or severe $(n, \%)$ & $14,36.8$ & $9,45.0$ & $5,27.8$ & \\
\hline No sexual intercourse $(n, \%)$ & $4,10.5$ & $2,10.0$ & $2,11.1$ & \\
\hline
\end{tabular}

Behçet's disease is defined as a systemic vasculitis at which activation of $\mathrm{T}$ lymphocytes and neutrophils, and increased oxidative stress have been reported to cause endothelial damage [2,11]. Endothelial damage and its indicator, EnD, have an important role in the pathophysiology of BD. On the other hand, EnD, which also plays a major role in the pathophysiology of ED, can be considered as the main component of the increased ED risk in the relatively young male population with BD (median age 34 years for this study). Accordingly, in this study, $4(80 \%)$ of 5 patients with ED were shown to have also EnD $(C F R<2)$. Various studies in the literature demon- 
strated the importance of EnD in the pathophysiology of ED [15]. Erectile dysfunction was suggested to be an early indicator of systemic vascular diseases and EnD, and the earliest clinical finding, similar as the tip of the iceberg [16]. In recent publications, ED has been reported to be an independent predictive factor for future cardiovascular diseases and researchers even have emphasized that it is an important clinical indicator for risk stratification in middle-aged men [15].

The association of BD with EnD has been demonstrated in a large number of clinical studies comparing the BD patients with healthy volunteers. The presence of EnD was also shown in patients without vascular involvement just as in patients with vascular involvement [17-21]. Oflaz et al. found that brachial artery FMD (flowmediated dilation) was significantly lower in patients with vascular involvement than in those with no vascular involvement, and they reported that there was no significant difference between the two groups in terms of carotid artery intima media thickness measurements [17]. In another study, Kayıkçığlu et al. found that homocysteine levels that are used as a bio-indicator of EnD were significantly higher in patients with vascular involvement compared with the levels of patients with no vascular involvement. The authors reported that endothelium-dependent dilatation was lower in patients with vascular involvement but their finding was not statistically significant [18]. Ulusoy et al. reported similar findings in a study comparing mucocutaneous BD patients with healthy volunteers. The authors found impaired EnD in $38 \%$ of MBD patients by using the brachial artery FMD method [19]. However, to our knowledge, there is no study that compared MBD and SBD patients in terms of EnD. In the present study, EnD was detected as $52.6 \%$ in MBD patients, and as $52.9 \%$ in SBD patients and there was no significant difference between the two groups $(p=0.985)$. Although the mucocutaneous group has better and more favourable prognosis, this finding showed that the MBD group had EnD risk similar to the patients with systemic involvement. We think that this is an interesting finding of this study which should be questioned and investigated in further studies.

However, we highlight the difference between MBD and SBD with respect to BD duration and the use of immunosuppressive agents. We think that these parameters may be related with a similar EnD risk in two groups. In this study, when compared with the MBD group, the use of immunosuppressive agents was found to be higher (0\% vs. $70.6 \%, p<0.001)$ in the SBD group. Since EnD and $E D$ rates were similar between the MBD and SBD groups, this finding can be interpreted that the use of immunosuppressive agents may reverse EnD and ED in SBD patients. Accordingly, immunosuppressive agents and tumour necrosis factor- $\alpha$ (TNF- $\alpha$ ) inhibitors have been reported to recover EnD in both $\mathrm{BD}$ and systemic vasculitis [21-24]. In a study, Protogerou et al. investigated the
Table 4. The details of patients with erectile dysfunction

\begin{tabular}{lccc}
\hline Patient number & BD subgroup & ED type & CFR value \\
\hline 4 & Vascular BD & Arterial & 1.29 \\
\hline 6 & Mucocutaneous BD & Venous & 1.28 \\
\hline 22 & Mucocutaneous BD & Arterial & 2.82 \\
\hline 26 & Vascular BD & Venous & 1.7 \\
\hline 31 & Neurological BD & Arterial & 1.1 \\
\hline
\end{tabular}

$B D$ - Behcet's disease, ED - erectile dysfunction, CFR-coronary flow reserve.

effect of disease activity (acute/active or stable/inactive) and corticosteroid use on endothelial function in BD [21]. The case-control cross sectional design part of this study showed that non-use of corticosteroids in active BD had significantly worse endothelial function compared to healthy controls $(p=0.007)$ whereas receiving corticosteroids in active BD had better endothelial function similar to healthy controls. In the prospective design part of the study, treatment with prednisolone (20 mg/day) in active BD significantly restored EnD within 7 days in BD patients without a history of previous corticosteroid use $(p=0.027)$. The authors also showed that endothelium independent vasodilatation was not significantly different either in the comparison between healthy controls and BD patients or in the comparison of pre- and post- prednisolone treatment in active BD [21]. In their study, Raza et al. reported that the suppression of acute inflammation using immunosuppressive agents (pulse cyclophosphamide plus methylprednisolone or continuous oral steroids) repaired EnD in primary systemic vasculitis [22]. The same group suggested that the use of infliximab which is a TNF- $\alpha$ inhibitor resulted rapid but transient improvement in EnD in primary systemic vasculitis [23]. The immunosuppressive agents in our cohort were azathioprine in 9 patients, mycophenolate mofetil in 1 patient, infliximab in 1 patient and corticosteroid in 1 patient. However, the role of immunosuppressive agents in EnD outcomes may be elucidated by a prospective randomized trial. In addition, the time from the diagnosis was significantly longer (24 vs. 102 months, $p=0.021$ ) in the SBD group of our study. Although there is limited evidence on the relation between disease duration and EnD in our literature search, Ozdemir et al. reported a finding of positive correlation between the duration of the disease and EnD in their study [24].

The above paragraph suggested that the use of immunosuppressive agents in the SBD group can be considered as a potential reducing factor for the risk of EnD whereas the duration of the disease can be considered as a potential factor increasing the risk of EnD.

Our study has some limitations. The small number of patients, the dissimilarity of treatment types and differences in treatment duration of the patients should be accepted as major limitations. However, comparative analysis of endothelial and erectile dysfunction in BD us- 
ing the objective quantitative methods (CFR and Penile Doppler USG) in mucocutaneous and systemic BD groups can be shown as the strengths of the study. On the other hand, the fact that the patient population in this study consisted of relatively younger males is an important point to emphasize in terms of ED risk.

\section{Conclusions}

In the present study we showed that, similar to systemic $B D$, the incidence of EnD and ED increases in the mucocutaneous BD patients. On the basis of EnD, it should be kept in mind that BD may lead to ED in men at an early age.

\section{Conflict of interest}

The authors declare no conflict of interest.

\section{References}

1. Yurdakul S, Yazici H. Behçet's syndrome. Best Pract Res Clin Rheumatol 2008; 22: 793-809.

2. Alpsoy E. Behçet's disease: a comprehensive review with a focus on epidemiology, etiology and clinical features, and management of mucocutaneous lesions. Br J Dermatol 2016; 43: 620-32.

3. Alpsoy E, Donmez L, Onder M, et al. Clinical features and natural course of Behcet's disease in 661 cases: a multicentre study. Clinical and Laboratory Investigations. Br J Dermatol 2007; 157: 901-6.

4. Sarica-Kucukoglu R, Akdag-Kose A, Kayabal M, et al. Vascular involvement in Behçet's disease: a retrospective analysis of 2319 cases. Int J Dermatol 2006; 45: 919-21.

5. Ehrlich GE. Vasculitis in Behçet's disease. Int Rev Immunol 1997; 14: 81-8.

6. Serdaroglu P, Yazici H, Ozdemir C, et al. Neurological involvement in Behçet's syndrome - a prospective study. Arch Neurol 1989; 46: 265-9.

7. Al-Araji A, Kidd DP. Neuro-Behçet's disease: epidemiology, clinical characteristics, and management. Lancet Neurol 2009; 8: 192-204.

8. NIH Consensus Conference. Impotence. NIH Consensus Development Panel on Impotence. JAMA 1993; 270: 83-90.

9. Hatzimouratidis K, Giuliano F, Moncada I, et al. European Associaiton of Urology Guidelines on Male Sexual Dysfunction. http://uroweb.org/guideline/male-sexualdysfunction/\#note_27

10. Gratzke C, Angulo J, Chitaley K, et al. Anatomy, physiology, and pathophysiology of erectile dysfunction. J Sex Med 2010; 7: 445-75.

11. Chambers JC, Haskard DO, Kooner JS. Vascular endothelial function and oxidative stress mechanisms in patients with Behçet's syndrome. J Am Coll Cardiol 2001; 37: 517-20.

12. Erdoğru T, Koçak T, Serdaroğlu P, et al. Evaluation and therapeutic approaches of voiding and erectile dysfunction in neurological Behçet's syndrome. J Urol 1999; 162: 147-53.

13. Aksu K, Keser G, Günaydin G, et al. Erectile dysfunction in Behçet's disease without neurological involvement: two case reports. Rheumatology 2000; 39: 1429-31.

14. International study group for Behçet's disease. Criteria diagnosis of Behçet's disease. Lancet 1990; 335: 1078-80.
15. Uddin SMI, Mirbolouk M, Dardari Z, et al. Erectile dysfunction as an independent predictor of future cardiovascular events: the multi-ethnic study of atherosclerosis. Circulation 2018; 138: 540-2.

16. Montorsi P, Montorsi F, Schulman CC. Is erectile dysfunction the "tip of the iceberg" of a systemic vascular disorder? Eur Urol 2003; 44: 352-4.

17. Oflaz H, Mercanoglu F, Karaman O, et al. Impaired endothelium-dependent flow-mediated dilation in Behçet's disease: more prominent endothelial dysfunction in patients with vascular involvement. Int J Clin Pract 2005; 59: 777-81.

18. Kayikçioğlu M, Aksu K, Hasdemir C, et al. Endothelial functions in Behçet's disease. Rheumatol Int 2006; 26: 304-8.

19. Ulusoy RE, Karabudak O, Kilicaslan F, et al. Noninvasive assessment of impaired endothelial dysfunction in mucocutaneous Behçet's disease. Rheumatol Int 2008; 28: 617-21.

20. Ozuguz P, Karabulut AA, Tulmac M, et al. Markers of endothelial dysfunction and evaluation of vascular reactivity tests in Behçet disease. Angiology 2014; 65: 937-43.

21. Protogerou AD, Sfikakis PP, Stamatelopoulos KS, et al. Interrelated modulation of endothelial function in Behcet's disease by clinical activity and corticosteroid treatment. Arthritis Res Ther 2007; 9: R90.

22. Raza K, Thambyrajah J, Townend JN, et al. Suppression of inflammation in primary systemic vasculitis restores vascular endothelial function: lessons for atherosclerotic disease? Circulation 2000; 102: 1470-2.

23. Raza K, Carruthers DM, Stevens R, et al. Infliximab leads to a rapid but transient improvement in endothelial function in patients with primary systemic vasculitis. Ann Rheum Dis 2006; 65: 946-8.

24. Ozdemir R, Barutcu I, Sezgin AT, et al. Vascular endothelium function and plasma homocysteine levels in Behcet's disease. Am J Cardiol 2004; 94: 522-5. 